\title{
Modelling and Fabrication of Geiger mode Avalanche Photodiodes ${ }^{1}$
}

\author{
W.J. Kindt ${ }^{2}$ and H.W. van Zeijl ${ }^{3}$ \\ ${ }^{2}$ Faculty of Information Technology and Systems, Department of Electrical Engineering, Delft University of Technology \\ Mekelweg 4, 2628 CD, Delft, The Netherlands \\ ${ }^{3}$ DIMES, Delft University of Technology, Feldmannweg 17, 2628 CT, Delft, The Netherlands
}

\begin{abstract}
As a first assessment for the fabrication of Geiger mode avalanche photodiode arrays, single pixel devices have been made. A CMOS compatible technology is used to allow the future integration of pixels in an array with readout electronics. A model for afterpulsing is presented that relates the afterpulsing probability to the concentration and capture cross section of the traps in the depletion layer. The bias voltage and temperature dependence of the dark count rate is explained by a trap assisted tunneling model. Measured results on fabricated devices are compared with theory.
\end{abstract}

\section{INTRODUCTION}

Avalanche Photodiodes (APDs) can be operated in the so called Geiger mode to count single photons. In the Geiger mode, the APD is biased above its breakdown voltage. If a photon is absorbed in the diode and generates an electron-hole pair, an avalanche breakdown will occur. After breakdown, the current is quenched and the diode is recharged for the detection of another photon.

Because of their large quantum efficiency, Geiger mode APDs, are excellent devices for measuring the photon flux of weak optical signals. They also have excellent time resolution, and are often used in photon timing measurements.

Little work has been done in the field of Geiger mode avalanche photodiode arrays (GAPAs), although there would be many applications for such devices [1]. A two-dimensional GAPA could be used for faint light level photon counting imaging, with, for example, applications in astronomy. A one dimensional GAPA could be used for photon timing measurements with spectral sensitivity, for instance for spectral-resolved measurements of fast fluorescent decays.

The main problem involved with the creation of GAPAs is optical cross talk between the pixels of the array. During breakdown a pixel may emit a few photons which can be detected by neighboring pixels. This could be prevented by an optical isolation between neighboring pixels, e.g. trenches filled with an opaque material [2].

The purpose of our work is to create integrated arrays of Geiger mode APDs. The first step towards this goal is the creation of individual pixels. For the creation of pixels, we paid special attention to two points.

The first point is the area efficiency of the pixel structure. To prevent edge breakdown, APDs often have a diffused guard ring around the cathode. The guard ring occupies an appreciable surface area and photons incident on this area will not be detect-

\footnotetext{
${ }^{1}$ This project was supported by the Technology Foundation (STW) under project no. DEL 44.3294
}

ed. The area efficiency of the pixel can be increased if the high field area beneath the cathode is defined by an additional anode implantation. This method also ensures that breakdown will occur in the bulk material and not at the edges of the junction [3].

The second point is that the pixels of a GAPA should be realized in a CMOS compatible technology. In a two-dimensional array a MOS transistor must be integrated with each pixel to allow readout of the entire array through $x$-y lines. In a one dimensional array the integration of readout electronics, such as active quenching circuits [4], is also desirable.

The fabrication of Geiger mode APDs is difficult because the devices are extremely sensitive to defects. Generation centers in the depletion layer of the diode cause thermal generation of electron-hole pairs. These will be detected as if they were generated by a photon, resulting in dark counts. Trapping centers in the space charge region may trap some of the charge carriers generated during the avalanche discharge. If these carriers are released after the APD has been recharged, they will also initiate a breakdown pulse. This effect is called afterpulsing.

To investigate the fabrication technology of Geiger mode avalanche photodiode arrays, we have fabricated single pixels. Edge breakdown in the pixel structure is prevented using an anode implantation through a window that is slightly smaller than the cathode area to obtain a large area efficiency. A polysilicon cathode is used for CMOS compatibility.

This paper first describes some results of the modeling of afterpulsing and dark counts. The afterpulsing model relates the afterpulsing probability to material parameters and predicts the bias voltage dependence of the afterpulsing probability. The dark count model explains the temperature and bias voltage dependence of the dark count rate. After this theoretical investigation, the fabrication technology used to create the APS is described. Eperimental results are presented and compared to theory.

\section{AFTERPULSING}

The afterpulsing model presented here consists of two parts. In the first part, the probability that a charge carrier will be trapped during a Geiger discharge and successfully initiate an afterpulse later is calculated. The second part describes the time dependence of the afterpulsing probability and relates the parameters of the first part of the model to quantities that can be measured.

In practical devices, different types of traps, each with different concentrations, capture cross sections and lifetimes are present. Some of the trapis capture holes and others electrons. First, a device with only one electron trap will be considered. 


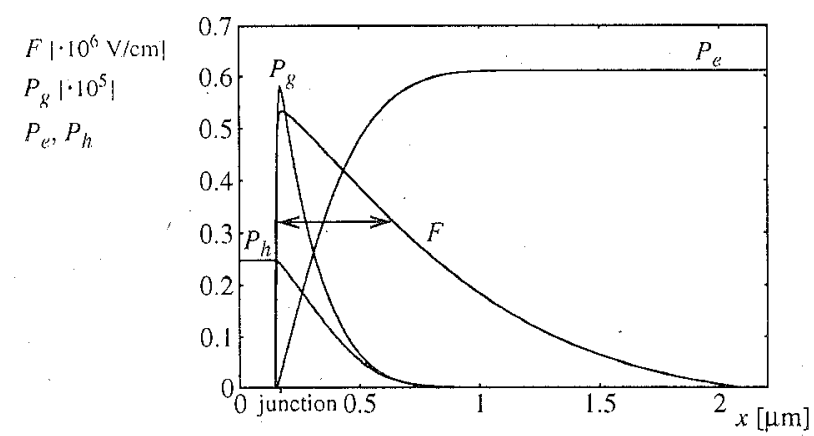

Figure 1: Electric field $(F)$, normalized generation probability distribution at breakdown $\left(P_{g}\right)$ and electron $\left(P_{e}\right)$ and hole $\left(P_{h}\right)$ avalanche initiation probabilities in a Geiger mode APD.

Figure 1 shows the field profile of an APD. This field profile was calculated for a doping profile that resulted from a process simulation. The high field region, indicated by the arrows, is the region where the field strength is large enough for impact ionization to occur. The boundaries of this region are not exactly defined, but the ionization probability decreases rapidly with decreasing field strength. During breakdown, many electronhole pairs are generated in this region. The generated electrons will flow to the n-type side of the junction (the left), and the generated holes to the p-type side of the junction (the right). The probability that one electron generated at position $x_{\xi}$, is trapped and causes an afterpulse later equals:

$$
P_{a l e}=\int_{x_{n}}^{x_{g}} N_{t e} \sigma_{t e} P_{e}(x) d x
$$

in which $N_{t e}$ is the electron trap concentration, $\sigma_{t e}$ is the electron trap cross section, $x_{n}$ is the position of the depletion layer boundary in the n-type material and $P_{e}(x)$ is the probability that an electron released in the depletion layer at position $x$ will initiate breakdown. $P_{e}(x)$ was calculated for the field profile of figure 1 using the method described in [5]. The result is also presented in figure 1, together with the hole avalanche initiation probability, $P_{h}$. The probability that a random electron generated somewhere in the Geiger discharge initiates breakdown is:

$$
P_{a e}=\int_{x_{n}}^{x_{p}} P_{g}\left(x_{g^{\prime}}\left(\int_{x_{n}}^{x_{g}} N_{t e} \sigma_{t e} P_{e}(x) d x\right) d x_{g}\right.
$$

in which $P_{g}(x)$ is the normalized sparial distribution of the ion ization events during breakdown. For simplicity, the high field region effective width for electron trap afterpulsing, $W_{e}$, is introduced, which simplifies (2) to:

$$
P_{a e}=N_{t e} \sigma_{t e} W_{e}
$$

For hole traps, a similar definition exists for $W_{h}$. To calculate $W_{e}$ and $W_{h}$, it is necessary to know $P_{g}$ during the Geiger discharge. A reasonable estimate for this function is the generation distribution at the breakdown voltage with a constant current through the diode. This distribution is also shown in figure 1.
Using this distribution, values of $W_{e}$ and $W_{h}$ were calculated. Resulting values are shown in figure 2 .

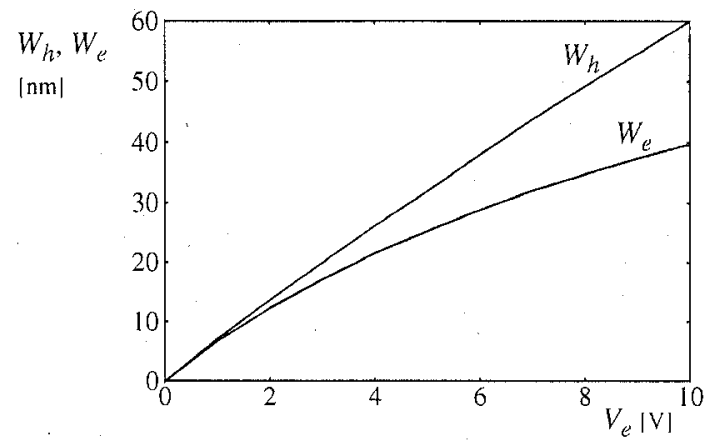

Figure 2: Calculated values for the high field region effective widths for electron and hole trapping, $W_{e}$ and $W_{h}$ as a function of the excess bias voltage $V_{e}$.

Given $P_{a e}$, the probability that one of the electrons generated during breakdown will be trapped and cause an afterpulse, the following equation follows for the total afterpulsing probability:

$$
P_{a t}=1-\left(1-N_{t e} \sigma_{t e} W_{e}\right)^{n} \approx N_{t e} \sigma_{t e} W_{e} n
$$

in which $n$ is the total number of electrons that is generated during breakdown.

The charge flowing through the depletion layer during a Geiger breakdown discharges the APD from $V_{b r}+V_{e}$ to $V_{b r}$. $V_{b r}$ is the breakdown voltage of the APD and $V_{e}$ the excess bias voltage, the amount of voltage the diode is operated above breakdown. Therefore:

$$
n=\frac{C V_{e}}{q}
$$

in which $C$ equals the depletion layer capacitance of the APD and $q$ is the electronic charge.

Combining (4) and (5) results in:

$$
P_{a t}=\frac{C}{q} N_{t e} \sigma_{t e} W_{e} V_{e}
$$

from which it follows that the afterpulsing probability increases with the area and the applied excess bias voltage. The increase with the bias voltage is superlinear because $W_{e}$ increases with the bias voltage. To minimize the afterpulsing probability, the stray capacitance and the capacitance of the readout circuitry should be as small as possible.

So far, only one type of trap has been considered. If different types of traps are present, each of them can be treated separately. The treatment of hole traps is analogous to that of electron traps. The total afterpulsing probability is the sum of the afterpulsing probabilities caused by the separate traps.

So far, only the probability that charge carriers will be captured by the traps during a breakdown pulse has been calculated. After the breakdown pulse, some of the traps may be filled and there will be a certain probability that a trapped carrier is released and causes an afterpulse. The probability density in time for the occurrence of an afterpulse after an initial Geiger dis- 
charge can be expressed as:

$$
P_{a}(t)=\sum_{i} A_{i} \exp \left(\frac{-t}{\tau_{i}}\right)
$$

Every term in this sum is caused by a different type of trap with its own concentration, cross section and lifetime $\tau_{i}$. Experimentally, $P_{a}(t)$ can be determined by measuring the autocorrelation function of the output signal of the APD [7]. By fitting (7) to the experimental results, the different $A_{i}$ and $\tau_{i}$ can be determined. From a measurement of $\tau_{i}$ versus temperature also the activation energy of the trap can be determined [6].

Equation (7) gives the afterpulsing probability as a function of time. The total afterpulsing probability is the integral of this quantity over time. For each trap, this probability equals the probability that the trap has been filled during the previous breakdown. Therefore, with (6):

$$
A_{i} \tau_{i}=\frac{C}{q} N_{t i} \sigma_{t i} W_{i} V_{e}
$$

in which $N_{t i}$ and $\sigma_{t e}$ are the concentration and cross section of the $\mathrm{i}^{\text {th }}$ trap concentration and $W_{i}$ equals $W_{e}$ or $W_{h}$, the high field region effective width for electron or hole trap afterpulsing, depending on the trap type.

\section{DARK COUNTS}

Dark counts are caused by the generation of e-h pairs in the depletion layer of the diode by other means than optical generation. Dark counts in Geiger mode APDs are analogous to the leakage current of conventional (photo)diodes. Usually the main cause of the leakage current is thermal generation in the depletion layer. Diffusion of minority carriers to the depletion layer edges can generally be neglected. Because Geiger mode APDs are operated at large electric field strengths, dark counts may also be caused by tunneling.

The large electric field strength in the depletion layer enhances the Shockley-Read-Hall generation rate due to trap assisted tunneling. This effect can be modeled with the trap assisted tunneling model [8], [9].

The dark count rate due to generation in the depletion layer equals:

$$
N_{d}=\int_{x_{n}}^{x_{p}} \frac{n_{i}}{\tau_{g}} P_{e h}(x) d x
$$

in which $n_{i}$ equals the intrinsic carrier concentration, and $P_{e h}$ is the probability that an $\mathrm{e}-\mathrm{h}$ pair generated at position $x$ will successfully initiate avalanche breakdown. $P_{e h}$ is equal to $P_{e}+P_{h}-P_{e} P_{h}$ (see figure 1). $\tau_{g}$ is the generation lifetime, which equals:

$$
\tau_{g}=\frac{\tau_{e 0}}{1+\Gamma} \exp \left(-\frac{E_{t}-E_{i}}{k T}\right)+\frac{\tau_{h 0}}{1+\Gamma} \exp \left(\frac{E_{t}-E_{i}}{k T}\right)=\frac{\tau_{g 0}}{1+\Gamma}
$$

in which $\tau_{e O}$ and $\tau_{h O}$ are the electron and hole recombination lifetimes, $E_{t}$ is the energy level of the dominant recombination center and $E_{i}$ is the intrinsic energy level. $\Gamma$ is the field effect function of the trap assisted tunneling model. $\tau_{g 0}$ is the generation lifetime at low electric field strengths. $\Gamma$ equals [8],[9]:

$$
\Gamma=2 \sqrt{3} \pi \frac{|F|}{F_{\Gamma}} \exp \left(\left(\frac{F}{F_{\Gamma}}\right)^{2}\right)
$$

in which $F$ is the local electric field strength and:

$$
F_{\Gamma}=\frac{\sqrt{24 m^{*}(k T)^{3}}}{q \bar{h}}
$$

in which $m^{*}$ is the effective mass for tunneling which equals $0.25 \cdot m_{0} \cdot m_{0}$ is the free electron mass, $k$ is the Boltzmann constant, $T$ is the absolute ternperature and $\hbar$ is the Dirac constant.

\section{PROTOTYPE FABRICATION}

Prototypes of Geiger mode APDs were fabricated in a $10 \Omega \mathrm{cm}$ p-type epilayer on a $\mathrm{p}^{+}$-type substrate. These wafers are normally used in a standard CMOS process at DIMES.

First the p-type high-field area is defined by a $150 \mathrm{keV}$ boron implantation. The dose of this implantation is used to control the breakdown voltage. With a dose of $6 \cdot 10^{12} \mathrm{~cm}^{-2}$, a breakdown voltage of $33 \mathrm{~V}$ results. The anode implantation is followed by a drive in at $1100^{\circ} \mathrm{C}$ for two hours in an inert environment. The drive in increases the breakdown voltage homogeneity. After the drive in, a boron implantation is performed to create substrate contacts. This implantation is annealed during the following cathode processing.

The process used to create the cathode can easily be combined with a CMOS process. First, a field oxide is grown. Subsequently this oxide is removed in windows where the cathode is to be formed. $200 \mathrm{~nm}$ polysilicon is deposited and implanted with arsenic (40keV $5 \cdot 10^{15} \mathrm{~cm}^{-2}$ ). The polysilicon outside the cathode areas is removed. This is followed by an anneal during which part of the arsenic diffuses into the crystalline silicon. A $300 \mathrm{~nm}$ LPCVD-TEOS oxide is deposited over the wafer, followed by the creation of contact holes to $\mathrm{n}$ and $\mathrm{p}$ regions and metallization.

To investigate the influence of the processing on the device characteristics, two variations of the process were used. Prior to the process sequence, on some wafers an intrinsic gettering step was performed. The oxygen in the bulk material was precipitated to form gettering sites during a temperature step at $750^{\circ} \mathrm{C}$ for six hours. On other wafers, instead of depositing an oxide over the cathode, a part of the polysilicon was oxidized. This results in a thinner polysilicon layer over the photodiodes and enhances the blue response of the A.PDs.

Devices with different areas were fabricated. The results presented in the next section were all obtained on diodes with an anode high field area of $46^{*} 46 \mu \mathrm{m}^{2}$.

To enable cooling of the APDs, they were glued to a small copper plate mounted in a hole in a printed circuit board. Bond wires connect the bond flaps of the dies to the board. The resulting devices can be mounted on a Peltier element inside a vacuum chamber. 


\section{EXPERIMENTAL RESULTS}

To measure the afterpulsing probability, the time at which Geiger discharges occurred were measured using a TTL circuit consisting of a $10 \mathrm{MHz}$ timer and a memory bank. Control logic in the circuit stored the time at which pulses occurred. After a certain data collection time, the local memory of the circuit was read out by a personal computer that calculated the autocorrelation function and afterpulsing probability. Figure 3 shows an example of the afterpulsing probability versus time.

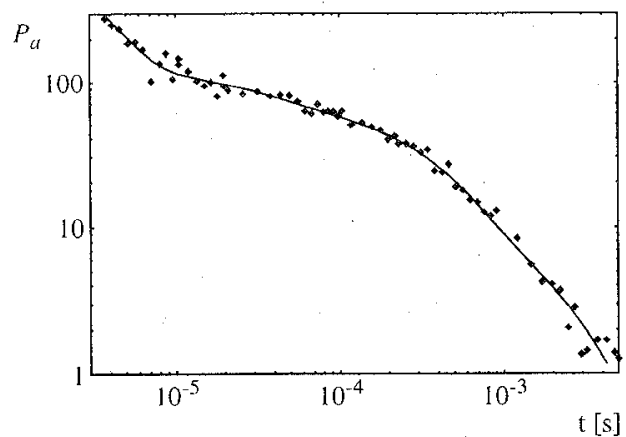

Figure 3: Afterpulsing probability vs. time, measured result and fitted curve.

A sum of exponential decay terms (see (7)) was fitted to the measured afterpulsing probability. Three different trap levels could clearly be distinguished in the measurement results. At high temperatures an additional trap, with a long lifetime, was detected and at low temperatures another trap with a short lifetime was observed. More traps with shorter or longer lifetimes may also be present, but they cannot yet be detected by our measurement system, which so far has used passive quenching. Also, traps with a very low concentration or very small capture cross section cannot be detected.

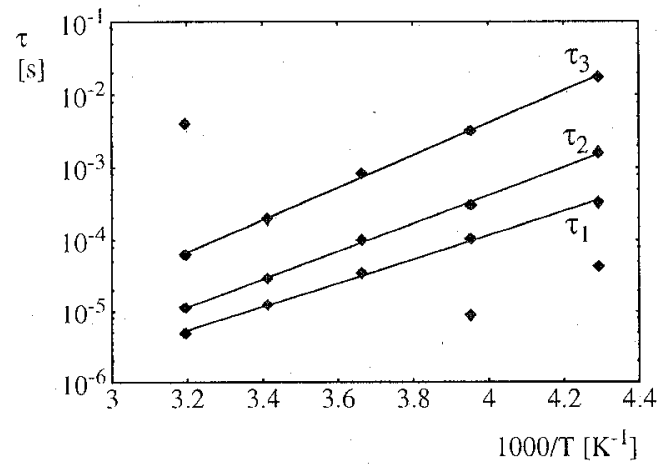

Figure 4: Trap decay times vs. temperature, measured values and fitted curves.

Figure 4 shows an Arrhenius plot of the trap decay times, $\tau_{i}$, that were determined by the fitting procedure. By fitting straight lines in this graph, the activation energies of the traps were determined at $0.33,0.39$ and $0.44 \mathrm{eV}$.

Table 1 gives the afterpulsing probabilities, $P_{a t i}=A_{i} \tau_{i}$, caused by the three individual trapping centers and the total afterpulsing probability measured at $5 \mathrm{~V}$ above breakdown and at a temperature of $20^{\circ} \mathrm{C}$.
Table 1: Partial and total afterpulsing probability

\begin{tabular}{|c|c|c|c|c|}
\hline$T\left({ }^{\circ} \mathrm{C}\right)$ & $P_{a t 1}(\%)$ & $P_{a t 2}(\%)$ & $P_{a t 3}(\%)$ & $P_{a, t o t}$ \\
\hline 40 & 0.8 & 8.2 & 2.3 & 11.3 \\
\hline 20 & 2.2 & 6.3 & 1.7 & 10.2 \\
\hline 0 & 3.1 & 3.5 & 0.7 & 7.3 \\
\hline-20 & 2.7 & 2.2 & 0.6 & 5.5 \\
\hline-40 & 1.8 & 1.8 & 1.6 & 5.2 \\
\hline
\end{tabular}

Figure 5 shows the measured afterpulsing due to the individual traps, $P_{a t i}$, as a function of the bias voltage at $20^{\circ} \mathrm{C}$. Also shown in this figure is the result of a calculation of the afterpulsing probability using (8). The figure shows a reasonable agreement between the afterpulsing model and measurement results. A value of $2.8 \mathrm{pF}$ was measured for the total capacitance of the diode, bondflaps, bondwires and packaging. Using this value for the capacitance, values of $3.0 \cdot 10^{-4}, 1.2 \cdot 10^{-4}$ and $6.5 \cdot 10^{-5} \mathrm{~cm}^{-1}$ had to be used for the product $N_{t} \sigma_{t}$ to obtain a reasonable fit. The best fit is obtained if in (8) $W_{e}$ is used for $P_{a t l}$ and $P_{a t 2}$ and $W_{h}$ for $P_{a t 3}$. This indicates that traps 1 and 2 are electron traps and trap 3 is a hole trap. A further improvement of the fit can be obtained if the bias voltage dependence of the depletion layer capacitance is taken into account. This will reduce the calculated values at larger bias voltages. Also, passive quenching was used during the experiments which resulted in a relatively large dead time. We expect that the use of an active quenching circuit will also improve the agreement between the measured data and calculated values.

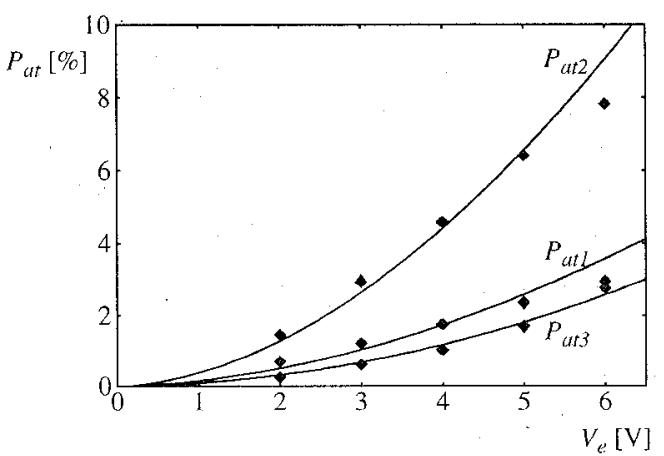

Figure 5: Measured and calculated afterpulsing probabilities due to two separate traps as a function of $V_{e}$.

Figure 6 shows the measured dark count rates for some devices measured at room temperature and at $-40^{\circ} \mathrm{C}$. The spread in the measurement results is probably caused by fluctuations in the generation center concentration. At room temperature the dark count rate is relatively large due to the rather large area of the diodes $\left(46^{*} 46 \mu \mathrm{m}^{2}\right)$. Very low dark count rates are obtained even with these large areas at $-40^{\circ} \mathrm{C}$. Analysis of the measurement results indicates that neither the gettering step nor the thermal oxidation of the top layer of polysilicon has a significant influence the dark count rate. 


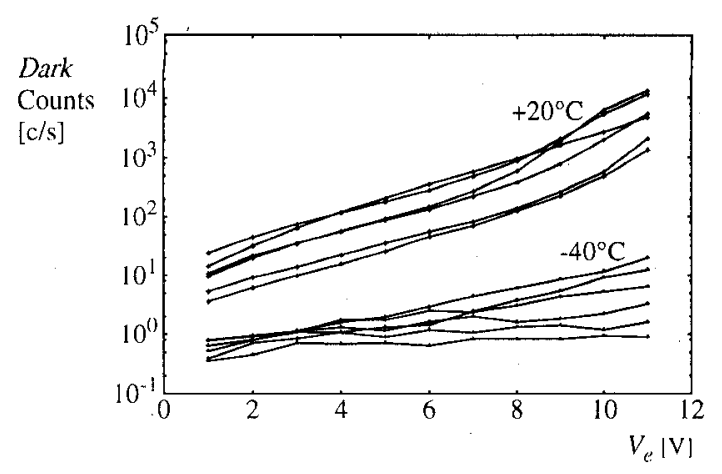

Figure 6: Measured dark count rates at different devices at $+20^{\circ} \mathrm{C}$ and $-40^{\circ} \mathrm{C}$.

Previous devices with implanted cathodes had a much larger dark count rate [3] than these devices with polysilicon cathodes. Our preliminary explanation is that the polysilicon material itself has acted as a getter and has effectively removed defects from the junction area. Future experiments will test this hypothesis.

Finally, figure 7 compares the measured dark count rates with calculated results using the trap assisted tunneling model (9)-(12). The doping profile used in the simulation was obtained from a Suprem [10] process simulation. The anode implantation dose used in the simulation was adjusted to $4.5 \cdot 10^{12} \mathrm{~cm}^{-2}$ to match the measured breakdown voltage. A generation lifetime of $34 \mathrm{~ms}$ was used in the calculations. The deviation between the measurements and calculations for larger temperatures and bias voltages will be investigated in the near future. Considering that only one fitting parameter $\left(\tau_{g 0}\right)$ has been used, the fit is reasonable. The trap assisted tunneling model therefore seems promising for the explanation of the bias voltage and temperature dependence of the dark count rate in Geiger mode APDs.

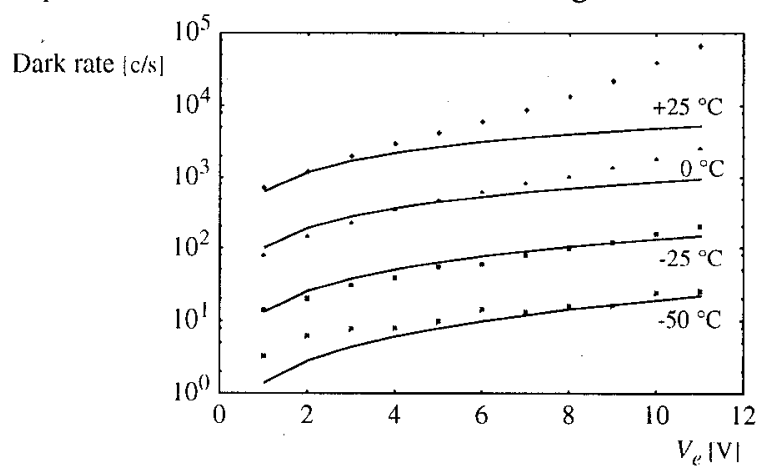

Figure 7: Measured and calculated dark count rates as a function of $V_{e}$ for different temperatures.

\section{CONCLUSIONS}

In this paper we have presented a new model for afterpulsing in Geiger mode avalanche photodiodes. The model relates the afterpulsing probability to the trap concentration and capture cross section and explains the bias voltage dependence of the afterpulsing probability. The trap assisted tunneling model, originally developed for diodes fabricated in modern, low voltage IC processes, has been applied to Geiger mode APDs. This model gives an explanation for the bias voltage and temperature dependence of the dark count rate. To allow the future realiza- tion of an integrated array of Geiger mode APDs, a CMOS compatible pixel for such an array has been developed. This pixel uses a polysilicon cathode. With this technology we have succeeded in the creation of APDs with a good behavior. Measurements on experimental devices agree reasonably with theoretical models.

\section{ACKNOWLEDGMENTS}

The authors would like to thank $W$. van der Vlist for the quick service he provided with the bonding of the devices and A.A. Bellekom and F. Creemer for their helpful remarks on the manuscript of this paper.

\section{REFERENCES}

[1] F. Zappa et al., "Integrated array of avalanche photodiodes for single-photon counting", Proceedings of the $27^{\text {th }}$ European solid-state device research conference, Stuttgart, Germany, Sep. 22-24, 1997, pp. 600-603.

[2] A. Mathewson, J.C. A.lderman, J. Ryan, R.M. Redfern and G.T. Wrixon, "Photon counting arrays for spatially varying, low light level signals", Proc. of SPIE, Vol. 2022, Photodetectors and power meters. 1993, pp. 312-143.

[3] W.J. Kindt, N.H. Shahrjerdy and H.W. van Zeijl, "A silicon avalanche photodiode for single optical photon counting in the Geiger mode", Sensors and Actuators, vol. A60, pp.98102, 1997.

[4] S. Cova, A. Longoni and G. Ripamonti, "Active quenching and gating circuits for single-photon avalanche diodes (SPADs)", IEEE Transactions on nuclear science, vol. 29, no. 1, Feb 1982, pp. 599-601.

[5] W.G. Oldham, R.R. Samuelson and P. Antognetti, "Triggering phonomena in avalanche diodes", IEEE Transactions on electron devices, vol. ED-19, no. 9, Sep 1972, pp. 1056-1060.

[6] S. Cova, A. Lacaita and G. Ripamonti, "Trapping phenomena in avalanche photodiodes on nanosecond scale", IEEE Electron device letters, vol. 12, no. 12, Dec. 1991, pp. 685687.

[7] H.Z. Cummins and E.R. Pike, Eds, "Photon correlation and light beating spectroscopy", Plenum, London, 1974, pp. 151-224.

[8] G.A.M. Hurkx, D.B.M. Klaassen and M.P.G. Knuvers, "A new recombination model for device simulation including tunneling", IEEE Transactions on electron devices, vol. ED-39, no. 2, Feb 1992, pp. 331-338.

[9] G.A.M. Hurkx, H.C. de Graaff, W.J. Kloosterman and M.P.G. Knuvers, "A new analytical diode model including tunneling and avalanche breakdown", IEEE Transactions on electron devices, vol. ED-39, no. 9, Sep 1992, pp. 20902098.

[10] Technology Modeling Associates, Inc., TSUPREM-4 twodimensional process simulation program. 